\title{
SIMULACION DEL MICROCLIMA EN UN INVERNADERO USADO PARA LA PRODUCCIÓN DE ROSAS BAJO CONDICIONES DE CLIMA INTERTROPICAL
}

\section{MICROCLIMATE I SIMULATION IN A GREENHOUSE USED FOR ROSES PRODUCTION UNDER CONDITIONS OF INTERTROPICAL CLIMATE}

\author{
Edwin Andrés Villagrán Munar ${ }^{1,2 *}$, y Carlos Ricardo Bojacá Aldana ${ }^{2}$ \\ ${ }^{1}$ Corporación Universitaria Minuto de Dios, Facultad de Ingeniería, Sede Cundinamarca, Av. 15 No \\ 1-22 Sur Barrio La Fragüita, Zipaquirá, Colombia. \\ ${ }^{2}$ Universidad Jorge Tadeo Lozano, Facultad de Ciencias Naturales e Ingeniería, Carrera 4 No. 22-61, \\ Módulo 6, Bogotá, Colombia. \\ * Autor para correspondencia E-mail: edwina.villagranm@utadeo.edu.co
}

\section{RESUMEN}

En Colombia, la producción de rosa (Rosa sp.) se desarrolla en un alto porcentaje en invernaderos ventilados en forma natural. El objetivo de este trabajo consistió en desarrollar una caracterización del microclima generado en el interior de un invernadero a través de la modelación numérica de los flujos de aire mediante un software CFD (Computational Fluids Dynamics) de volúmenes finitos. El modelo CFD-3D utilizado fue validado mediante recopilación de datos experimentales, y se compararon datos experimentales y simulados, los que mostraron un grado de ajuste adecuado y una misma tendencia para las variables de temperatura y humedad relativa. Los resultados obtenidos muestran que para condiciones de clima diurno el microclima generado se encuentra en rangos óptimos para la producción de rosa. Para condiciones de clima nocturno, se observó una presencia constante del fenómeno de inversión térmica del aire interior del invernadero, lo cual genera condiciones subóptimas para el crecimiento y desarrollo de las plantas.

Palabras clave: invernadero, simulación CFD, temperatura, humedad relativa, periodo diurno, periodo nocturno.

\section{ABSTRACT}

In Colombia, roses (Rosa sp.) are mostly cultivated in naturally ventilated greenhouses. The objective of this work was to develop a characterization of the microclimate generated inside a greenhouse through the numerical modelling of air flows by using the CFD (Computational Fluids Dynamics) software of finite volumes. The CFD-3D model used was validated through experimental data collection and the results obtained from the comparison of experimental and simulated data, which showed a suitable degree of adjustment and the same tendency for the variables of temperature and relative humidity. The results obtained show that, for daytime conditions, the microclimate generated is in optimal ranges for the production of rose. For night-time climate conditions, presence of thermal inversion phenomenon in the indoor air of the greenhouse was observed, which generates suboptimal conditions for the growth and development of plants.

Key words: greenhouse, CFD simulation, temperature, relative humidity, diurnal period, nocturnal period.

Recibido: 15 enero 2019. Aceptado: 20 marzo 2019. 


\section{INTRODUCCION}

Colombia lidera actualmente la producción de ornamentales y flores de corte en América del Sur (Mena-Vásconez et al., 2016). La rosa (Rosa sp.) es una de las especies más cultivadas y comercializadas en el mercado internacional. Esta especie se encuentra establecida en invernaderos de plástico, que desde hace varias décadas se convirtieron en la opción tecnológica predominante para el cultivo protegido en climas templados y cálidos (McCartney et al., 2018). En Colombia existe una amplia gama de diseños y tamaños de invernaderos que han evolucionado a partir del invernadero tradicional colombiano, siendo uno de éstos el invernadero multitúnel tipo millenium, aunque se debe resaltar que mantiene la característica de estructuras de bajo costo y ventiladas pasivamente (Villagran et al., 2018).

El microclima generado en estos invernaderos es dependiente del clima exterior. Al no contar con sistema de control de clima activo, el control de las variables ambientales relevantes para la producción agrícola se limita al intercambio de aire entre el exterior y el interior del invernadero a través de las áreas de ventilación dispuestas en la estructura. Esto ha generado que en las últimas décadas existan numerosos trabajos dedicados a la optimización y evaluación del fenómeno de ventilación natural en invernaderos (Baeza et al., 2009; Villagran et al., 2012; He et al., 2015).

El estudio del comportamiento microclimático de un invernadero puede realizarse a través de modelos empíricos de balance de energía, experimentación en campo, termografía digital o mediante simulación numérica aplicando técnicas como la dinámica de fluidos computacional (CFD, por sus siglas en inglés) (Chen, 2009). Esta herramienta permite realizar el análisis cualitativo y cuantitativo del comportamiento de variables de interés, como temperatura, humedad y concentración de especies en estructuras agrícolas o pecuarias bajo diferentes escenarios de simulación (Bournet and Boulard, 2010). Un estudio mediante modelos CFD se compone de tres fases de desarrollo: (i) el pre-proceso, fase donde se genera la geometría y el mallado del invernadero; se establecen las condiciones de frontera y se seleccionan las principales propiedades físicas del fluido en estudio; (ii) la fase del proceso, en la cual se definen los modelos numéricos a utilizar y la forma de discretización de las ecuaciones parciales no lineales que se involucran en este tipo de estudios, y es la fase donde se busca la convergencia del problema alcanzando así una solución; y (iii) la fase de postproceso, donde los usuarios pueden observar los resultados numéricos y gráficos obtenidos en la geometría estudiada; esta fase suministra la información necesaria para realizar la calibración y validación del modelo CFD utilizado (EspinelMontes et al., 2015).

El objetivo de este trabajo consistió en desarrollar y ajustar un modelo numérico CFD $3 \mathrm{D}$, con el fin de estudiar el comportamiento de las variables temperatura y humedad y su relación con la ventilación natural en un invernadero multitúnel tipo millenium colombiano bajo condiciones climáticas de la sabana de Bogotá, usado para la producción de rosa.

\section{MATERIALES Y MÉTODOS}

\section{Sitio y descripción del invernadero}

El trabajo experimental se desarrolló en un invernadero comercial de plástico tipo multitúnel millenium de $5610 \mathrm{~m}^{2}$ de área cubierta, perteneciente a una finca productora de rosas $\left(4^{\circ} 35^{\prime} 32,44^{\prime \prime}\right.$ N; 74ำ1 '35,14' W; 2510 msnm) ubicada en el municipio de El Rosal, Cundinamarca, Colombia. El invernadero evaluado estaba compuesto de 15 naves, cada una con una luz de 6,9 m (Fig.1A), las alturas mínimas y máximas bajo canal fueron de 3,0 y $8,2 \mathrm{~m}$, respectivamente, la distancia longitudinal del invernadero era de $55 \mathrm{~m}$ y estaba orientado en sentido este-oeste (E-W). Cada nave disponía de un área de ventilación cenital fija de 0,53 m de apertura; el área total de ventilación natural se complementaba con aperturas de 2,0 $\mathrm{m}$ en los cuatro costados de la estructura, para un total de área de ventilación de $856 \mathrm{~m}^{2}$.

Se evaluaron las condiciones meteorológicas exteriores para las 24 horas del día, dentro del periodo comprendido entre las 00:00 horas del 05 de junio y las 23:00 horas de 06 de julio del año 2016. El registro de las variables se realizó por medio de una estación meteorológica (Vantage Pro2 Plus, Davis Instruments, Hayward, California, USA), que integraba sensores de radiación global, temperatura, humedad relativa, precipitación, velocidad y dirección del viento; la frecuencia de registro era cada 10 minutos.

\section{Simulación numérica}

Las ecuaciones gobernantes de un flujo pueden ser representadas como ecuaciones de convección-difusión de un fluido para tres leyes de conservación, que incluyen las ecuaciones de momento, energía y transporte de un fluido compresible y en un campo tridimensional (3D) en estado estacionario:

$$
\frac{\partial \rho \phi}{\partial t}+\nabla(\rho \phi \vec{v})=\nabla(\Gamma \nabla \phi)+\mathrm{S}
$$

donde $\rho$ es la densidad del fluido $\left(\mathrm{kg} \mathrm{m}^{-3}\right), \nabla$ es 

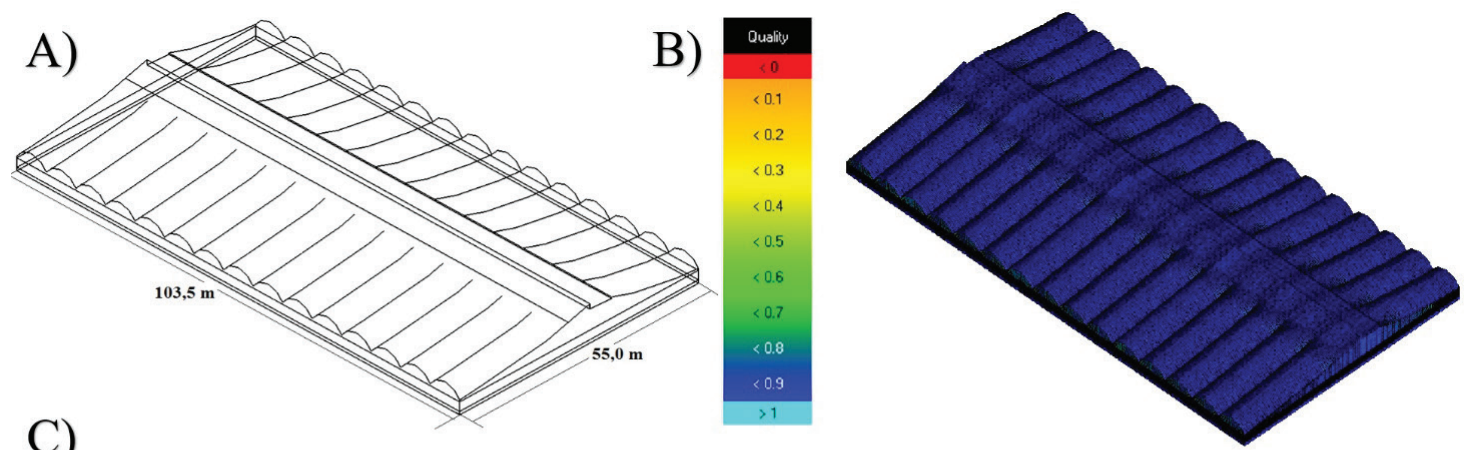

C)

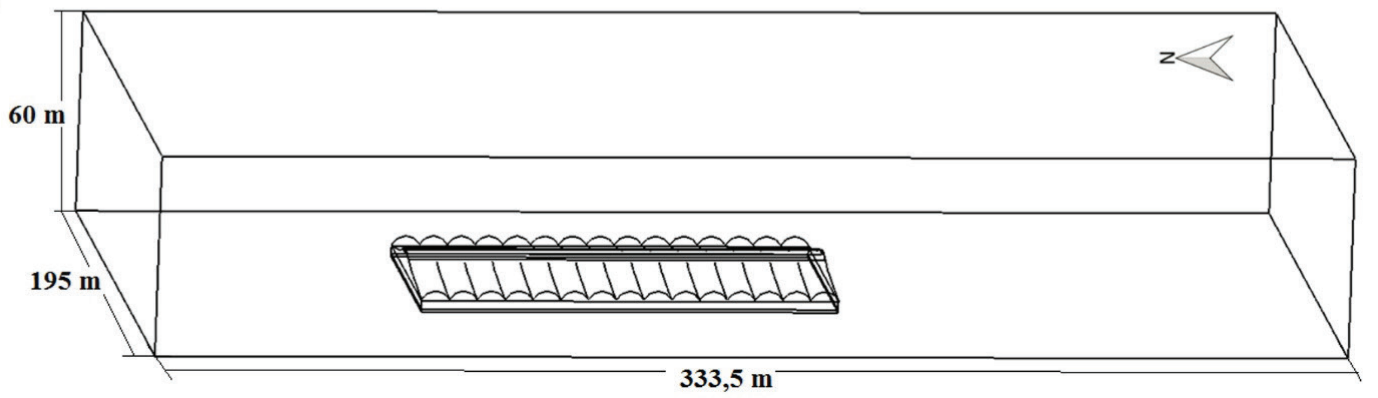

Fig. 1. A) Área del invernadero evaluado; B) detalle de la calidad de la malla; C) dominio computacional.

Fig. 1. A) Area of the greenhouse evaluated; B) details of the quality of the mesh; C) computational domain.

el operador nabla que es un operador vectorial que puede ser aplicado a un escalar o a un vector y representa su gradiente en el espacio, $\phi$ representa la variable de concentración, $\nabla$ es el vector de velocidad $\left(\mathrm{m} \mathrm{s}^{-1}\right), \Gamma$ es el coeficiente de difusión $\left(\mathrm{m}^{2} \mathrm{~s}^{-1}\right)$ y $S$ representa el término fuente (Piscia et al., 2012).

El comportamiento de la humedad y la conservación de masa fue modelado a partir de la siguiente expresión (Rojano et al., 2014):

$$
\nabla(\rho Y i)=-\nabla J_{1}+\mathrm{R}_{\mathrm{i}}+\mathrm{E}_{\mathrm{i}}
$$

donde $\mathrm{Y}$ es la fracción local de masa de cada especie a través de la solución de la ecuación convección-difusión, E es el termino fuente de humedad, J es el flujo de difusión de las especies, y $R_{i}$ es la tasa de producción de especies por componente i. Debido a que las velocidades de viento serán bajas en algunas zonas en el interior del invernadero, los efectos de flotabilidad influenciados por el cambio de densidad del aire estarán presentes (Espinel et al., 2015) y pueden ser modelados a través del modelo de Boussinesq de acuerdo con el trabajo desarrollado por Kacira et al. (2004), que se describe mediante la siguiente expresión:

$$
\begin{aligned}
& \left(\rho-\rho_{0}\right) g=-\rho_{0} \beta\left(\mathrm{T}-\mathrm{T}_{0}\right) \mathrm{g} \quad \mathrm{y} \\
& \beta=-\left(\frac{1}{\rho}\right)\left(\frac{\partial \rho}{\partial \mathrm{T}}\right)_{\mathrm{p}}=\frac{1}{\rho} \frac{\mathrm{p}}{\mathrm{RT}^{2}}=\frac{1}{\mathrm{~T}}
\end{aligned}
$$

Así mismo todos los escenarios simulados consideraron la ecuación energía y el modelo de radiación seleccionado fue el de ordenadas discretas (DO) con discretización angular. Este modelo permite realizar el análisis de clima en condiciones de periodo nocturno, simulando y resolviendo el fenómeno de radiación desde el suelo del invernadero hacia el ambiente exterior. Para tal fin se consideró el cielo como un cuerpo negro con una temperatura equivalente $\left(\mathrm{T}_{\mathrm{C}}\right)$ de $10^{\circ} \mathrm{C}$ para un escenario predominante de noches húmedas y nubadas (Iglesias et al., 2009).

Estos conjuntos de ecuaciones fueron resueltos mediante la utilización del software comercial ANSYS Fluent (v. 17.0) (Ansys Corporation, 2016), usando una malla no estructurada y el método de volúmenes finitos. La naturaleza turbulenta del flujo de aire se simuló utilizando el modelo de turbulencia estándar $k-\varepsilon$, modelo ampliamente usado y validado en estudios enfocados a invernaderos demostrando un ajuste y precisión adecuada (Piscia et al., 2015).

\section{Dominio computacional y generación de la malla}

El software de pre-proceso ANSYS ICEM CFD (v. 17.0) se utilizó para generar un gran dominio computacional compuesto por el invernadero y sus alrededores, esto con el fin de garantizar la no afectación de la solución numérica del campo de flujo fuera del invernadero, y para permitir una 
definición apropiada de la capa límite atmosférica (Rico-Garcia, 2011). Las dimensiones del dominio computacional fueron de 333,5; 195 y $60 \mathrm{~m}$ para los ejes $\mathrm{x}, \mathrm{y}, \mathrm{z}$, respectivamente (Fig. 1C). Este tamaño se determinó siguiendo las pautas para el cálculo de CFD del entorno eólico alrededor de los edificios (Tominaga et al., 2008).

El dominio computacional se dividió en $56,190,701$ volúmenes discretizados en el espacio, número de elementos que se obtuvo luego de verificar la independencia de las soluciones numéricas del flujo de aire a tamaños de malla con un número superior e inferior de elementos de acuerdo al procedimiento reportado por $\mathrm{He}$ et al. (2017). Un criterio fundamental para establecer la precisión de las soluciones obtenidas mediante CFD consiste en evaluar la calidad de la malla, este procedimiento se realiza desde la interfaz del software de pre-proceso (Fig. 1B). Los parámetros de calidad evaluados fueron el tamaño de las celdas y la variación del tamaño de celda a celda encontrando que un 90,5\% de las celdas de la malla estaban dentro del intervalo de calidad alta (0,95-1). También se evaluó el ortogonal quality, donde el valor mínimo obtenido fue de 0,88, resultados que se clasifican dentro del rango de alta calidad (Flores-Velázquez et al., 2015).

El software comercial de procesamiento CFD ANSYS FLUENT (v. 17.0) se utilizó para realizar las simulaciones. El método de solución semi implícito para la ecuación de presión (SIMPLE) y el algoritmo de resolución de partición de presión implícita con la división de operadores (PISO) fue aplicado para resolver el campo de flujo del fluido simulado. Los criterios de convergencia del modelo fueron establecidos en $10^{-8}$ para la ecuación de energía y en $10^{-6}$ para las ecuaciones de continuidad, momento y turbulencia (Baxevanou et al., 2017).

\section{Condiciones de frontera}

El límite superior del dominio y las superficies paralelas al flujo fueron fijados con condiciones de frontera de propiedades simétricas para no generar pérdidas de fricción del flujo de aire en contacto con estas superficies. Las simulaciones consideraron las características atmosféricas del sitio experimental, tales como presión atmosférica de $74993 \mathrm{~Pa}$, viscosidad del aire igual a $1,7 \mathrm{E}-05 \mathrm{~kg} \mathrm{~m}^{-1} \mathrm{~s}^{-1} \mathrm{y}$ gravedad de $9,81 \mathrm{~m} \mathrm{~s}^{-2}$. Al límite inferior y las paredes del invernadero se les fijó una condición de frontera de pared antideslizante. Otras propiedades del polietileno y del suelo agrícola como calor especifico $(C p)$, conductividad térmica $(k)$ y densidad $(\rho)$ fueron establecidas de acuerdo con Villagrán et al. (2012)

$$
\text { Los límites perimetrales del dominio }
$$

computacional se establecieron como límites de entrada de aire o salida de presión según el caso evaluar. Se consideró un perfil uniforme de velocidad del viento evaluando velocidades con valores entre 0,32 y $1,22 \mathrm{~m} \mathrm{~s}^{-1}$ y valores de temperatura medios para cada hora del día. Dichos valores fueron establecidos a partir de la información climática recopilada en el periodo de medición experimental.

Otros parámetros de entrada que son necesarios para alimentar el modelo de radiación son las propiedades ópticas del material de cubierta establecidas para el fenómeno de radiación infrarroja de onda larga. Los valores para el polietileno transparente fueron coeficiente absorción $(\alpha)$ de 0,69 , coeficiente de transmisión $(T)$ de 0,19 y coeficiente de reflexión $(\rho)$ de 0,11 . El modelo no incluyó cultivo alguno y adicionalmente se asumió una hermeticidad máxima del invernadero. Estas simplificaciones son válidas puesto que son aplicadas a cada una de los casos simulados y los errores que puedan derivarse tendrán el mismo grado de magnitud para cada escenario.

\section{Escenarios considerados}

La evaluación del comportamiento micro climático se realizó estableciendo como condiciones de entrada al modelo CFD, el valor de los parámetros meteorológicos medios obtenidos para cada hora evaluada (Tabla 1) y la configuración de ventilación estándar del invernadero estudiado, para este caso, fue ventilación lateral combinada con ventilación de techo para horas diurnas y ventilación de techo para las horas nocturnas, para cada simulación se calculó el Índice de renovación (IR) de acuerdo con la siguiente expresión:

$$
I R=\frac{\mathrm{Q}}{V_{i}} * 3600
$$

donde IR es el índice de renovación horario ( $\mathrm{Vol}$ $\left.h^{-1}\right), Q$ es el caudal de flujo de aire por unidad de tiempo $\left(\mathrm{m}^{3} \mathrm{~s}^{-1}\right)$ en las áreas de ventilación, es el volumen del invernadero $\left(\mathrm{m}^{3}\right)$.

\section{Validación del modelo desarrollado}

La temperatura de bulbo seco y de bulbo húmedo del aire del invernadero se registró por medio de treinta termopares tipo $\mathrm{T}$ (cobreConstantán) conectados a un número igual de registradores de datos (Cox-Tracer Junior, Escort DLS, Edison, New Jersey, USA). Los conjuntos de termopares se desplegaron uniformemente a lo largo del eje transversal y longitudinal del invernadero a una altura de 1,5 m sobre el nivel del suelo y se programaron para registrar datos con una frecuencia de 10 minutos, datos que 
Tabla 1. Condiciones meteorológicas medias usadas como parámetros de entrada al modelo CFD-3D. Table 1. Average meteorological conditions used as input parameters to the CFD-3D model.

\begin{tabular}{|c|c|c|c|c|c|}
\hline Hora & $\begin{array}{c}\text { Temperatura } \\
\left({ }^{\circ} \mathrm{C}\right)\end{array}$ & $\begin{array}{l}\text { Velocidad del } \\
\text { viento } \\
\left(\mathrm{m} \mathrm{s}^{-1}\right)\end{array}$ & $\begin{array}{c}\text { Dirección } \\
\text { del viento } \\
\left({ }^{\circ}\right)\end{array}$ & $\begin{array}{c}\text { Humedad } \\
\text { relativa } \\
(\%)\end{array}$ & $\begin{array}{c}\text { Radiación } \\
\text { solar } \\
\left(\mathrm{w} \mathrm{m}^{-2}\right)\end{array}$ \\
\hline 00:00 & 11,42 & 0,06 & 92,04 & 92,4 & 0 \\
\hline 01:00 & 11,13 & 0,04 & 96,92 & 92,8 & 0 \\
\hline 02:00 & 10,9 & 0,04 & 93,35 & 93,9 & 0 \\
\hline 03:00 & 10,7 & 0,05 & 93,41 & 93,7 & 0 \\
\hline 04:00 & 10,43 & 0,01 & 86,79 & 94 & 0 \\
\hline 05:00 & 10,08 & 0,01 & 89,6 & 94,1 & 0 \\
\hline 06:00 & 9,85 & 0,04 & 95,36 & 94,4 & 0,63 \\
\hline 07:00 & 10,31 & 0,08 & 99,23 & 90,2 & 62,67 \\
\hline 08:00 & 12,5 & 0,36 & 96,29 & 85,6 & 210,3 \\
\hline 09:00 & 14,04 & 0,73 & 99,75 & 80,3 & 346,33 \\
\hline 10:00 & 15,39 & 0,9 & 106,41 & 76,2 & 460 \\
\hline 11:00 & 16,33 & 1,01 & 109,41 & 75,1 & 488,7 \\
\hline $12: 00$ & 16,59 & 1,15 & 121,5 & 74,7 & 380,9 \\
\hline 13:00 & 16,81 & 1,22 & 116,5 & 73,2 & 411,9 \\
\hline 14:00 & 17,01 & 1 & 115,3 & 72,5 & 410,7 \\
\hline 15:00 & 17,09 & 0,82 & 108,3 & 71,7 & 345,3 \\
\hline $16: 00$ & 16,96 & 0,71 & 120,8 & 75 & 279,2 \\
\hline $17: 00$ & 16,32 & 0,66 & 123,5 & 77,4 & 186 \\
\hline 18:00 & 15,32 & 0,34 & 116,54 & 82,1 & 56,41 \\
\hline 19:00 & 14,04 & 0,13 & 109,3 & 83,9 & 0 \\
\hline 20:00 & 13,12 & 0,09 & 112,8 & 86,3 & 0 \\
\hline 21:00 & 12,51 & 0,06 & 94,94 & 87,7 & 0 \\
\hline 22:00 & 12,1 & 0,04 & 94,31 & 90 & 0 \\
\hline 23:00 & 11,79 & 0,05 & 93,14 & 91.4 & 0 \\
\hline
\end{tabular}

posteriormente fueron analizados y organizados en valores medios para cada hora, y mediante el uso de ecuaciones psicrométricas se determinó el valor de la humedad relativa. Los datos obtenidos en forma experimental serán comparados con los obtenidos mediante simulación CFD.

\section{RESULTADOS Y DISCUSIÓN}

\section{Validación del modelo}

La evolución temporal de la temperatura y la humedad relativa en el interior del invernadero se puede observar en la Fig. 2. Los órdenes en magnitud de los valores de temperatura simulados estuvieron de acuerdo con los medidos experimentalmente, se obtuvo un error cuadrático medio (MSE) de $0,17^{\circ} \mathrm{C}$, un error medio absoluto (MAE) de $0,36^{\circ} \mathrm{C}$ y un error porcentual absoluto medio (MAPE) de $2,54 \%$. Para el caso de la humedad relativa se encontró una tendencia similar entre los datos simulados y medidos, obteniendo un valor de MSE igual a 5,70\%, el MAE fue de 2,16\% y un MAPE de 2,67\%. En general se puede concluir que el ajuste del modelo CFD es adecuado ya que los errores encontrados están dentro de los rangos aceptables para los modelos de clima bajo invernadero (Baptista, 2007). Por lo tanto, este modelo CFD permitirá analizar de forma fiable el comportamiento de la temperatura y la humedad relativa del invernadero estudiado.

\section{Periodo diurno \\ Campo de flujo}

En la Fig. 3 se presentan los patrones de flujo de aire en el interior del invernadero para las 08:00, 12:00 y 16:00 horas. Estos flujos de aire son generados por el fenómeno de ventilación natural influenciados por la convección libre vía flotabilidad o efecto térmico y la convección forzada generada por el efecto eólico (Baeza et al., 2012). Para la hora 08:00 se observa un flujo que ingresa por la ventana frontal de barlovento con una velocidad media de 0,31 $\mathrm{m} \mathrm{s}^{-1}$ (Fig. 3A); este flujo de aire fresco desplaza el aire cálido del interiordelinvernaderogenerandounmovimiento convectivo que se dirige horizontalmente en el eje longitudinal del invernadero buscando la 

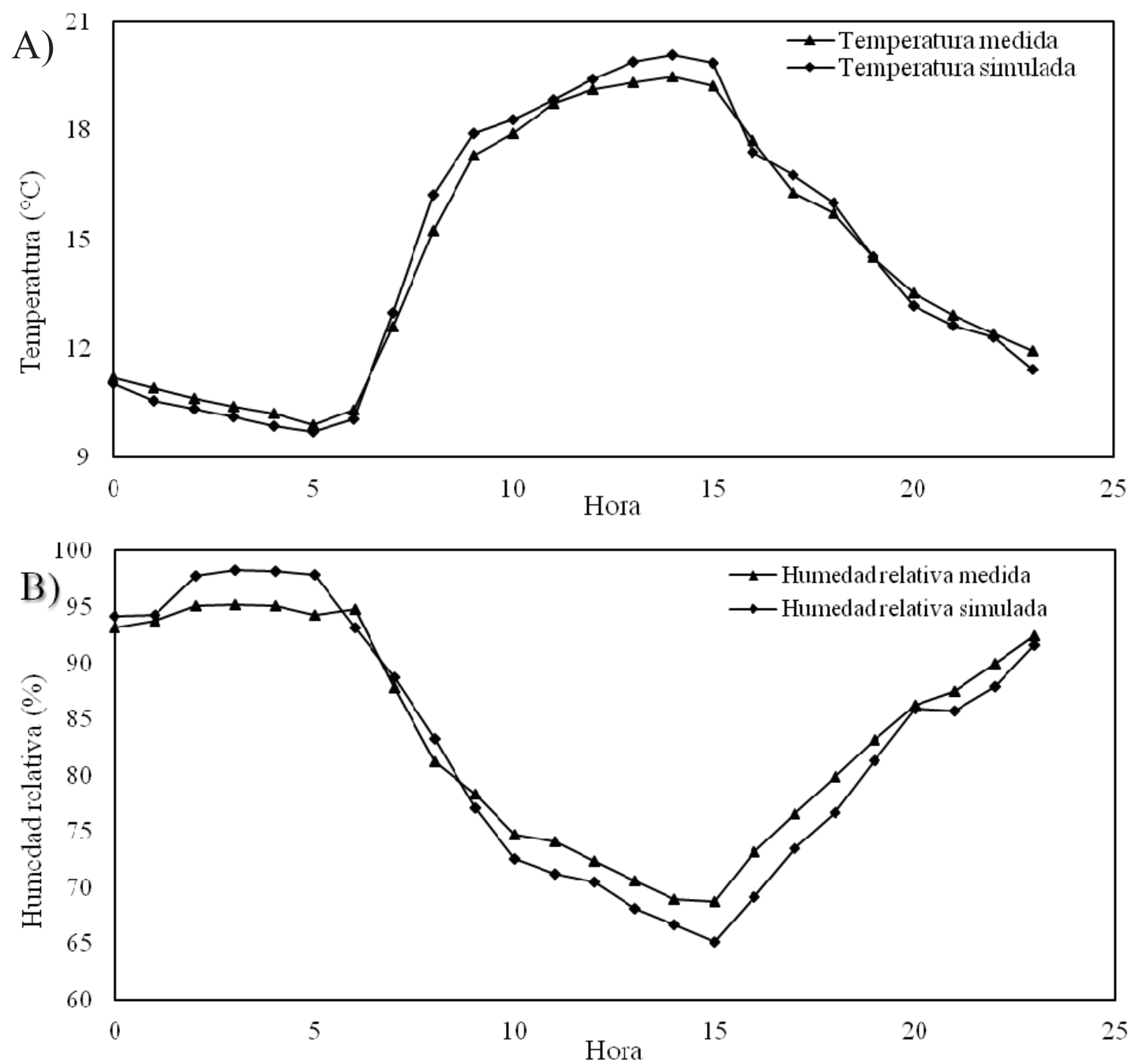

Fig. 2. A) Perfil de temperatura horario medido y simulado; B) Perfil de humedad relativa horaria medido y simulado dentro del invernadero evaluado.

Fig. 2. A) Measured and simulated temperature profile; B) Relative humidity profile measured and simulated within the greenhouse evaluated.

salida por la ventana frontal de sotavento, y en el eje vertical en dirección de la aperturas fijas de techo; este efecto combinado genera un Índice de Renovación (IR) de 7,3 vol h${ }^{-1}$. Para las horas 12:00 y 16:00 se observa un comportamiento similar con velocidades de 1,09 y $0,38 \mathrm{~m} \mathrm{~s}^{-1}$, respectivamente, generando unos IR de 26,31 y $11,31 \mathrm{vol} \mathrm{h}^{-1}$ para cada una de estas horas (Fig. 3B, C).

En la Tabla 2 se presentan los valores de IR para el periodo comprendido entre las 06:00 $\mathrm{y}$ las 18:00 horas. Se debe mencionar que para ninguna de estas horas evaluadas el IR cumple con el valor mínimo recomendado para invernaderos ventilados naturalmente, que debe ser igual a 40 vol h-1 (Montero et al., 2006). Los valores de IR moderadamente bajos pueden generar condiciones microclimáticas subóptimas y heterogéneas en el interior del invernadero. Adicionalmente se debe mencionar que los IR presentan una relación directa con los valores de velocidad de viento exterior y los diferenciales térmicos entre el interior y el exterior del invernadero $\left(\Delta T=T_{\text {media interior }}-T_{\text {media exterior }}\right)$, relación que fue previamente reportada por López et al. (2011).

\section{Comportamiento térmico}

La Fig. 4 muestra la distribución de la temperatura del aire en una vista isométrica a 1,5 m sobre el nivel del suelo para las 08:00, 12:00 y 16:00 horas. Las simulaciones permitieron observar que el valor de la temperatura para 


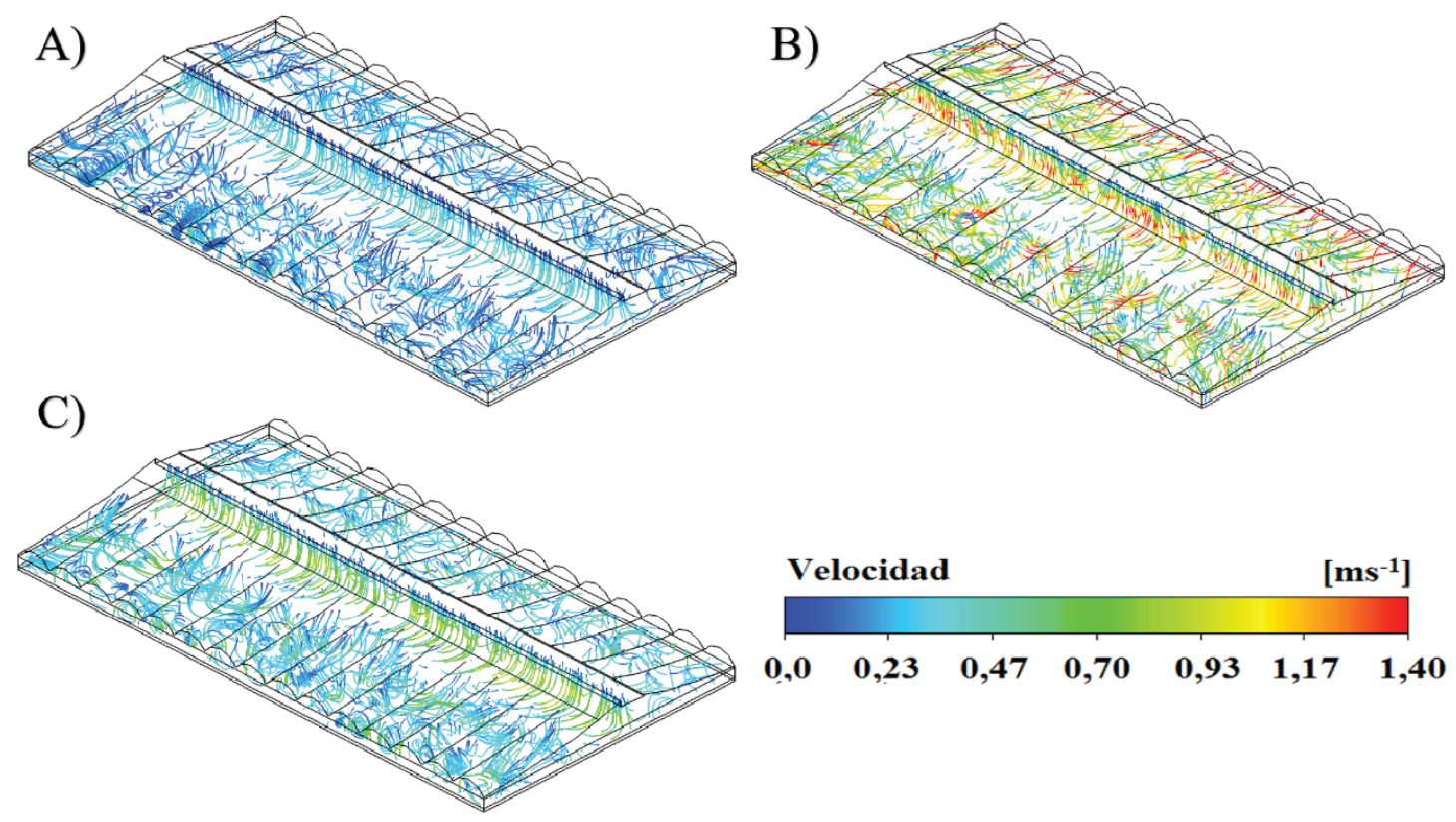

Fig. 3. Contornos de flujo de aire ( $\left.\mathrm{m} \mathrm{s}^{-1}\right)$ simulados para: A) hora 08:00; B) hora 12:00; C) hora 16:00. Fig. 3. Simulated airflow contours $\left(\mathrm{m} \mathrm{s}^{-1}\right)$ for: A) hour 08:00; B) hour 12:00; C) hour 16:00.

Tabla 2. Parámetros microclimáticos obtenidos para el periodo diurno.

Table 2. Microclimatic parameters obtained for the diurnal period (daytime).

\begin{tabular}{ccccc}
\hline Hora & $\begin{array}{c}\text { Temperatura } \\
\text { media interior } \\
\left({ }^{\circ} \mathbf{C}\right)\end{array}$ & $\begin{array}{c}\text { Diferencial } \\
\text { térmico } \\
\left(\boldsymbol{\Delta} \mathbf{T},{ }^{\circ} \mathbf{C}\right)\end{array}$ & $\begin{array}{c}\text { Humedad } \\
\text { relativa media } \\
\text { interior }(\%)\end{array}$ & $\begin{array}{c}\text { Índice de } \\
\text { renovación } \\
\left(\mathbf{V o l ~ h}^{-1}\right)\end{array}$ \\
\hline $06: 00$ & 10,05 & 0,2 & 93,1 & 1,1 \\
$07: 00$ & 12,96 & 2,65 & 88,7 & 1,4 \\
$08: 00$ & 16,21 & 3,71 & 83,2 & 7,3 \\
$09: 00$ & 17,9 & 3,86 & 77,1 & 16,7 \\
$10: 00$ & 18,31 & 2,92 & 72,5 & 21,6 \\
$11: 00$ & 18,85 & 2,52 & 71,2 & 23,54 \\
$12: 00$ & 19,41 & 2,82 & 70,5 & 26,31 \\
$13: 00$ & 19,89 & 3,08 & 68,1 & 27,89 \\
$14: 00$ & 20,09 & 3,08 & 66,7 & 25,62 \\
$15: 00$ & 19,87 & 2,78 & 65,1 & 17,56 \\
$16: 00$ & 17,41 & 0,45 & 69,1 & 11,21 \\
$17: 00$ & 16,78 & 0,46 & 73,5 & 10,54 \\
$18: 00$ & 16,01 & 0,69 & 76,6 & 5,34 \\
\hline
\end{tabular}

la hora 08:00 presenta un comportamiento heterogéneo con dos zonas marcadas desde la zona central del invernadero y los costados de este. La temperatura alcanzó un valor medio de $16,2^{\circ} \mathrm{C}$ y la diferencia entre el valor de temperatura máximo y mínimo fue de aproximadamente $0,93^{\circ} \mathrm{C}$ (Fig. 4A). El comportamiento es similar para las 12:00 y 16:00 horas (Fig. 4B, C) donde la temperatura media alcanzó valores de 19,41 y $17,41^{\circ} \mathrm{C}$, respectivamente, y las diferencias entre los valores máximos y mínimos de temperatura para cada caso son de 1,98 y $1,26^{\circ} \mathrm{C}$.

En la Tabla 2 se presentan los valores de temperatura media interior y $\Delta \mathrm{T}$ para las horas del periodo diurno; se debe destacar que estos valores son altamente dependientes de las condiciones presentadas en el ambiente exterior (Tabla 1) y principalmente del valor 

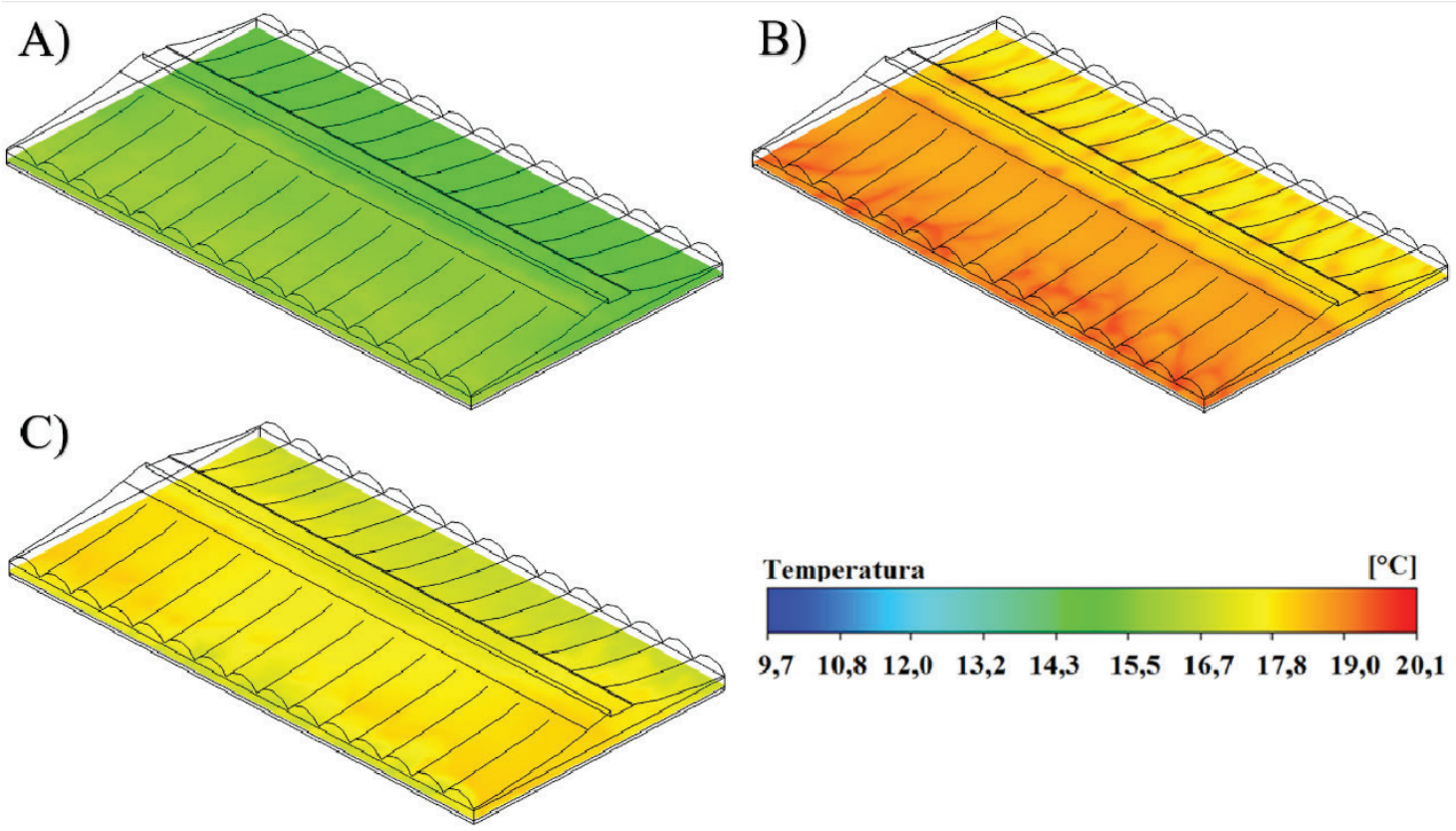

Fig. 4. Contornos de temperatura $\left({ }^{\circ} \mathrm{C}\right)$ simulados para: $\left.\mathrm{A}\right)$ hora 08:00; $\mathrm{B}$ ) hora 12:00; C) hora 16:00. Fig. 4. Temperature contours $\left({ }^{\circ} \mathrm{C}\right)$ simulated for: A) hour 08:00; B) hour 12:00; C) hour 16:00.

de la radiación. En general se observa que el invernadero exhibe condiciones térmicas aceptables para la producción de rosas en el intervalo comprendido entre las 09:00 y 16:00 horas, donde la temperatura se mantiene dentro de los rangos óptimos recomendados para este cultivo, los cuales deben oscilar entre 17 y $25^{\circ} \mathrm{C}$ (Kinfe et al., 2014) y preferiblemente entre 21 y $24^{\circ} \mathrm{C}$ (Yong, 2004). Lo anterior se traduce que bajo las condiciones presentadas en el invernadero los ciclos vegetativos pueden alargarse debido a que los valores de temperatura no superan los $21^{\circ} \mathrm{C}$. Esta situación puede ser compensada a través de un manejo adecuado de las áreas de ventilación, limitando el grado de apertura lo que permitiría aumentar la ganancia térmica del invernadero hasta generar las condiciones deseadas, aunque debe mencionarse que esta actividad debe ser realizada teniendo en cuenta los valores de humedad y $\mathrm{CO}_{2}$ presentes en el invernadero.

\section{Comportamiento de la humedad relativa}

El comportamiento de la Humedad Relativa (HR) se puede observar en la Fig. 5. La distribución espacial de HR a 1,5 m sobre el nivel del suelo muestra un comportamiento heterogéneo para las 08:00, 12:00 y 16:00 horas. El valor medio de HR obtenido para la hora 8:00 fue $83,2 \%$, bajo esta condición se encontraron zonas con valores máximos de HR de $87,3 \%$ y valores mínimos de $73,4 \%$ (Fig. $5 \mathrm{~A}$ ), al igual que en la variable temperatura este comportamiento no es recomendado para la producción agrícola, ya que se somete a las plantas a condiciones diferenciadas en un ambiente donde el manejo de las plantas se hace de forma homogénea sobre toda el área de cultivo establecida. Esto se traduce en una producción final heterogénea tanto en cantidad como en calidad. Por otro lado, el ataque de patógenos es más frecuente en las zonas donde las condiciones microclimáticas son más críticas. La HR muestra una relación inversa con la variable temperatura ya que las zonas de altas y bajas temperaturas concuerdan con las zonas de bajas y altas HR. Es importante mencionar que este comportamiento heterogéneo puede estar influenciado por los valores bajos de IR presentados para este invernadero. Para las horas 12:00 y 16:00 el comportamiento encontrado es similar al de la hora 08:00 y los valores medios de $\mathrm{HR}$ observados son de 70,5 y $69,1 \%$ respectivamente (Fig. 5B, C).

En la Tabla 2 se observan los valores de HR para las horas diurnas. Se puede mencionar que estos valores se encuentran en unos rangos óptimos para la producción de rosa en el periodo comprendido entre las 09:00 y 18:00 horas. Para las horas 06:00 y 07:00 la HR presenta valores superiores al $85 \%$, estas mismas horas presentan temperaturas inferiores a $15^{\circ} \mathrm{C}$; este comportamiento no es recomendado ya que puede inducir enfermedades en el follaje de la planta tales como mildiu velloso (Peronospora sparsa) y Botritys cinerea, enfermedades que en el 

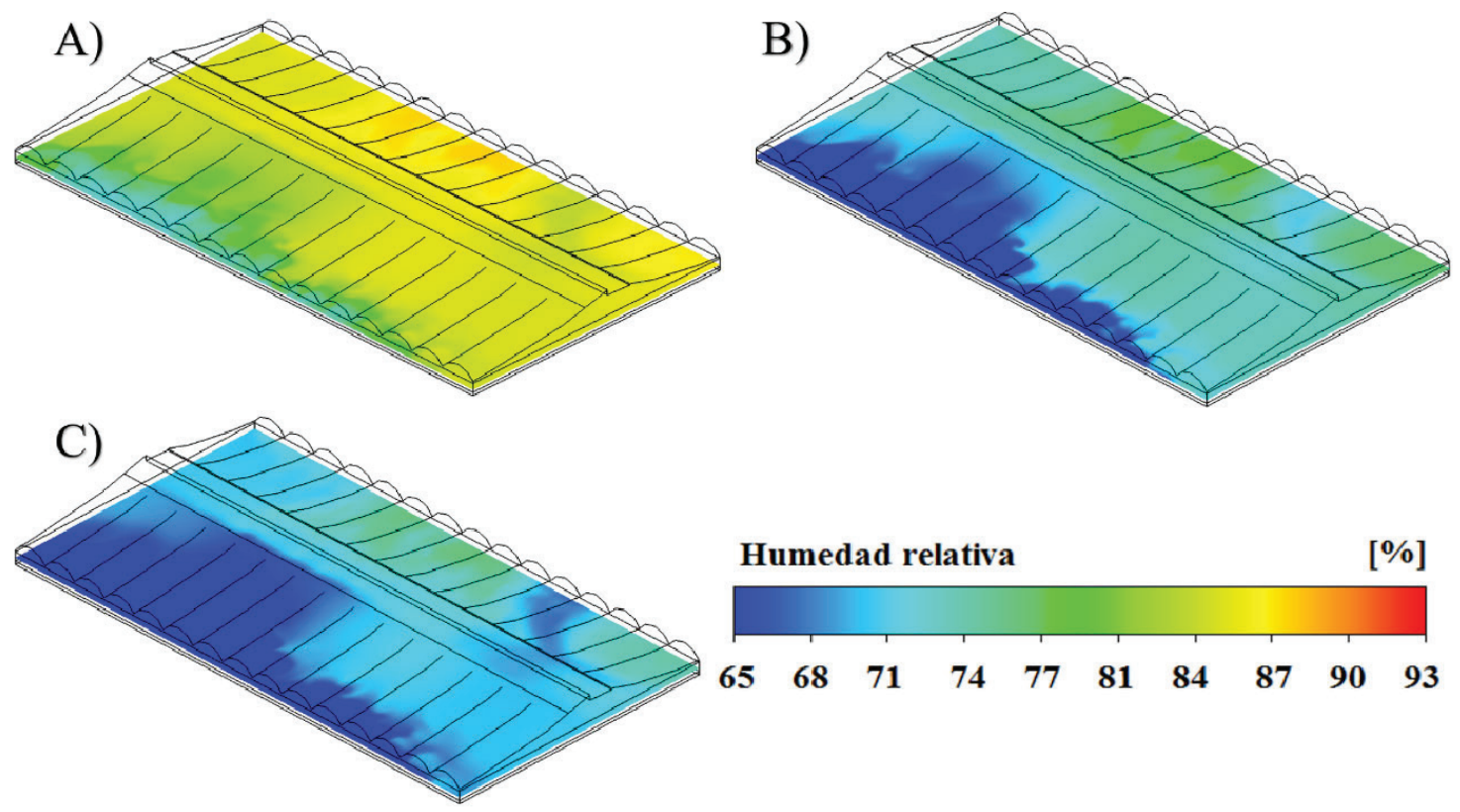

Humedad relativa

Fig. 5. Contornos de humedad relativa (\%) simulados para: A) hora 08:00; B) hora 12:00; C) hora 16:00. Fig. 5. Relative humidity contours (\%) simulated for: A) hour 08:00; B) hour 12:00; C) hour 16:00.

contexto colombiano son frecuentes y generan una alta pérdida económica, debido al uso intensivo de fungicidas para el manejo y control de dichas enfermedades (Castillo et al., 2010; Restrepo, 2010).

\section{Periodo nocturno \\ Campo de flujo}

La Fig. 6 muestra el comportamiento de los flujos de aire para las 20:00, 00:00 y 04:00 horas, bajo estas condiciones donde la velocidad exterior del viento es baja (Tabla 1) y las aperturas laterales de ventilación se encuentran cerradas. El movimiento del aire es inducido por el efecto térmico de la ventilación natural. Estos movimientos se caracterizan por ser de tipo convectivo rotatorio en dirección desde el suelo hacia la cubierta del invernadero. Este fenómeno ocurre debido a almacenamiento de calor por parte del suelo en las horas diurnas y su posterior liberación a la atmósfera en las horas nocturnas (Mesmoudi et al., 2017). Para la hora 20:00 pueden diferenciarse tres áreas de flujos convectivos donde la velocidad media del flujo de aire es de 0,28 $\mathrm{m} \mathrm{s}^{-1}$ (Fig. 6A). Para las horas 00:00 y 04:00, las velocidades de los flujos de aire están por debajo de los $0,1 \mathrm{~m} \mathrm{~s}^{-1}$ y se logran identificar movimientos convectivos en cada una de las naves que componen el invernadero (Fig. $6 \mathrm{~B}, \mathrm{C})$.

En la Tabla 3 se presentan los IR para las horas nocturnas evaluadas. En general estos
IR son bajos, ya que el intercambio de aire se realiza a través de las aperturas de techo la cuales permanecen abiertas y permiten el intercambio de aire entre el interior y el exterior del invernadero. Este movimiento de aire está fuertemente influenciado por el fenómeno de flotabilidad.

\section{Comportamiento térmico}

La temperatura bajo los escenarios simulados de condición nocturna presentó un comportamiento espacial homogéneo (Fig. 7). El valor medio de la temperatura para las 20:00, 00:00 y 04:00 horas, fue de 13,$15 ; 11,13 ;$ y $9,85^{\circ} \mathrm{C}$, respectivamente. Esta tendencia decreciente del valor de la temperatura es la que ocurre típicamente en los invernaderos pasivos que carecen de sistemas de calefacción.

En la Tabla 3 se presentan los valores medios de temperatura en el interior del invernadero para el periodo comprendido entre las 18:00-23:00 y las 00:00-05:00 horas. En general se observa que estos valores están por debajo del valor mínimo recomendado para la producción de rosas, que es de $16^{\circ} \mathrm{C}$; esta condición particular puede ocasionar desordenes fisiológicos en las plantas y flores de baja calidad comercial (Yong, 2004).

En la Tabla 3 se pueden encontrar los valores de $\Delta \mathrm{T}$. Los resultados reflejan que en el periodo comprendido entre las 23:00 y las 05:00 horas el valor de $\Delta \mathrm{T}$ es negativo. Esto es un indicativo que el invernadero se encuentra bajo condiciones de 


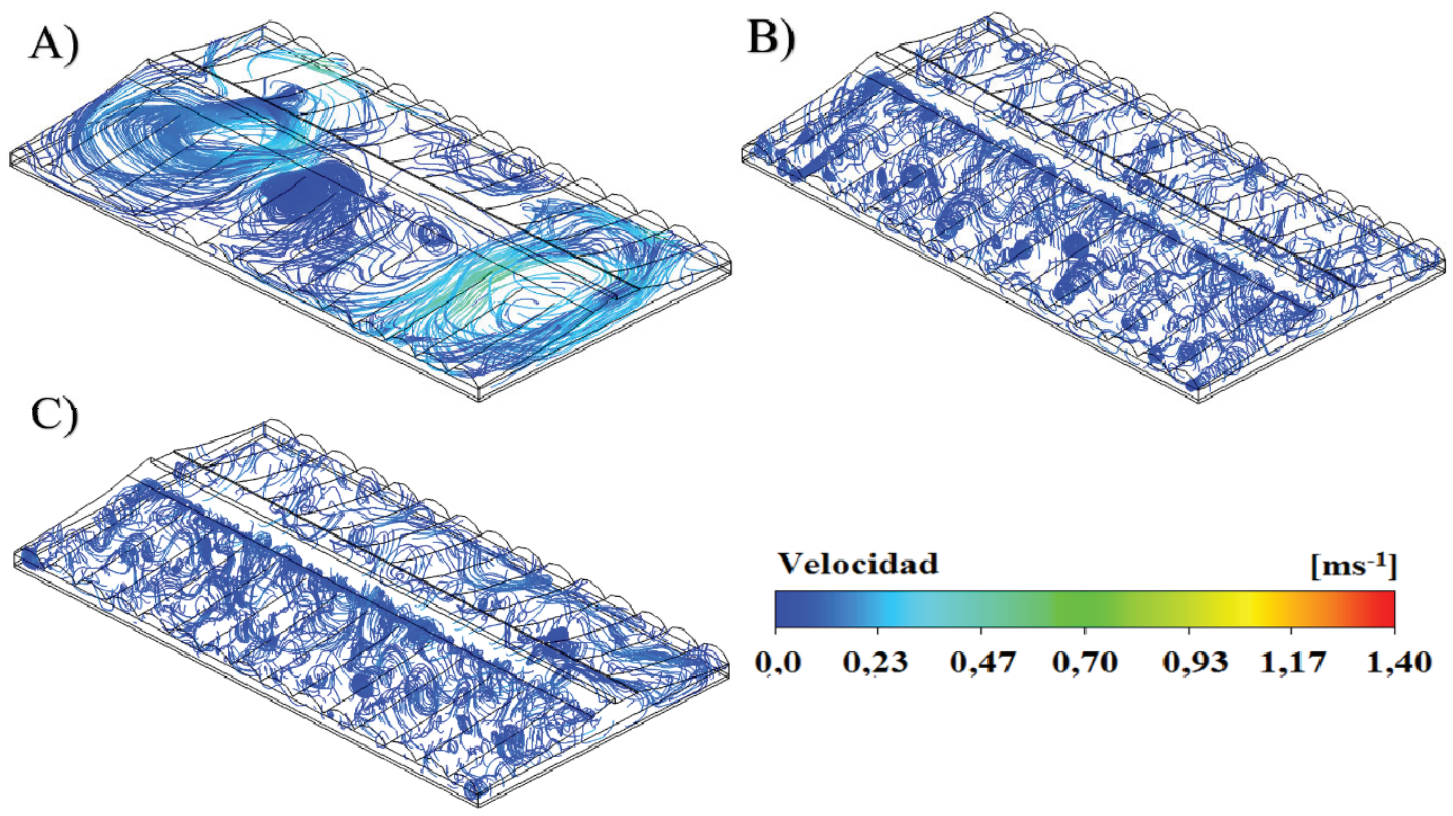

Fig. 6. Contornos de flujo de aire ( $\left.\mathrm{m} \mathrm{s}^{-1}\right)$ simulados para: A) hora 20:00; B) hora 00:00; C) hora 04:00. Fig. 6. Simulated airflow contours $\left(\mathrm{m} \mathrm{s}^{-1}\right)$ for: A) hour 20:00; B) hour 00:00; C) hour 04:00.

Tabla 3. Parámetros micro climáticos obtenidos para el periodo nocturno. Table 3. Microclimatic parameters obtained for the nocturnal period (night-time).

\begin{tabular}{ccccc}
\hline Hora & $\begin{array}{c}\text { Temperatura } \\
\text { media } \\
\text { interior }\left({ }^{\circ} \mathbf{C}\right)\end{array}$ & $\begin{array}{c}\text { Diferencial } \\
\text { térmico } \\
\left(\Delta \mathbf{T},{ }^{\circ} \mathbf{C}\right)\end{array}$ & $\begin{array}{c}\text { Humedad } \\
\text { relativa media } \\
\text { interior } \mathbf{( \% )}\end{array}$ & $\begin{array}{c}\text { Índice de } \\
\text { renovación } \\
\left(\mathbf{V o l ~ h}^{-1}\right)\end{array}$ \\
\hline 19:00 & 14,5 & 0,46 & 81,3 & 2,43 \\
20:00 & 13,15 & 0,03 & 85,9 & 1,57 \\
$21: 00$ & 12,61 & 0,1 & 85,7 & 1,12 \\
$22: 00$ & 12,31 & 0,21 & 87,9 & 0,67 \\
$23: 00$ & 11,42 & $-0,37$ & 91,6 & 0,68 \\
$00: 00$ & 11,13 & $-0,29$ & 94,1 & 0,86 \\
$01: 00$ & 10,56 & $-0,57$ & 94,3 & 0,57 \\
$02: 00$ & 10,31 & $-0,59$ & 97,8 & 0,67 \\
$03: 00$ & 10,09 & $-0,61$ & 98,3 & 1,2 \\
$04: 00$ & 9,85 & $-0,58$ & 98,2 & 1,3 \\
$05: 00$ & 9,7 & $-0,38$ & 97,9 & 0,97 \\
\hline
\end{tabular}

inversión térmica, fenómeno donde el ambiente en el interior del invernadero presenta un valor térmico inferior al del ambiente exterior. Esto se genera principalmente por una pérdida acelerada de calor a través de la cubierta y del flujo de calor extraído del interior del invernadero a través de las aperturas cenitales fijas (Montero et al., 2013). La presencia del fenómeno de inversión térmica no es recomendable en invernaderos usados para la producción agrícola, ya que las plantas estarán sometidas a condiciones térmicas subóptimas, que pueden limitar de forma parcial o total la producción de rosa. Por lo tanto, se sugiere la búsqueda de estrategias que permitan mejorar la hermeticidad del invernadero evaluado.

\section{Comportamiento de la humedad relativa}

La evolución espacial de la HR en el interior del invernadero para las horas 20:00, 00:00 y 04:00 se muestra en la Fig. 8. Se puede observar un comportamiento heterogéneo de esta variable climática; para la hora 08:00 el valor medio de la HR fue de $85,9 \%$, se observan zonas con valores mínimos y máximos de HR de 81 y $89 \%$, 


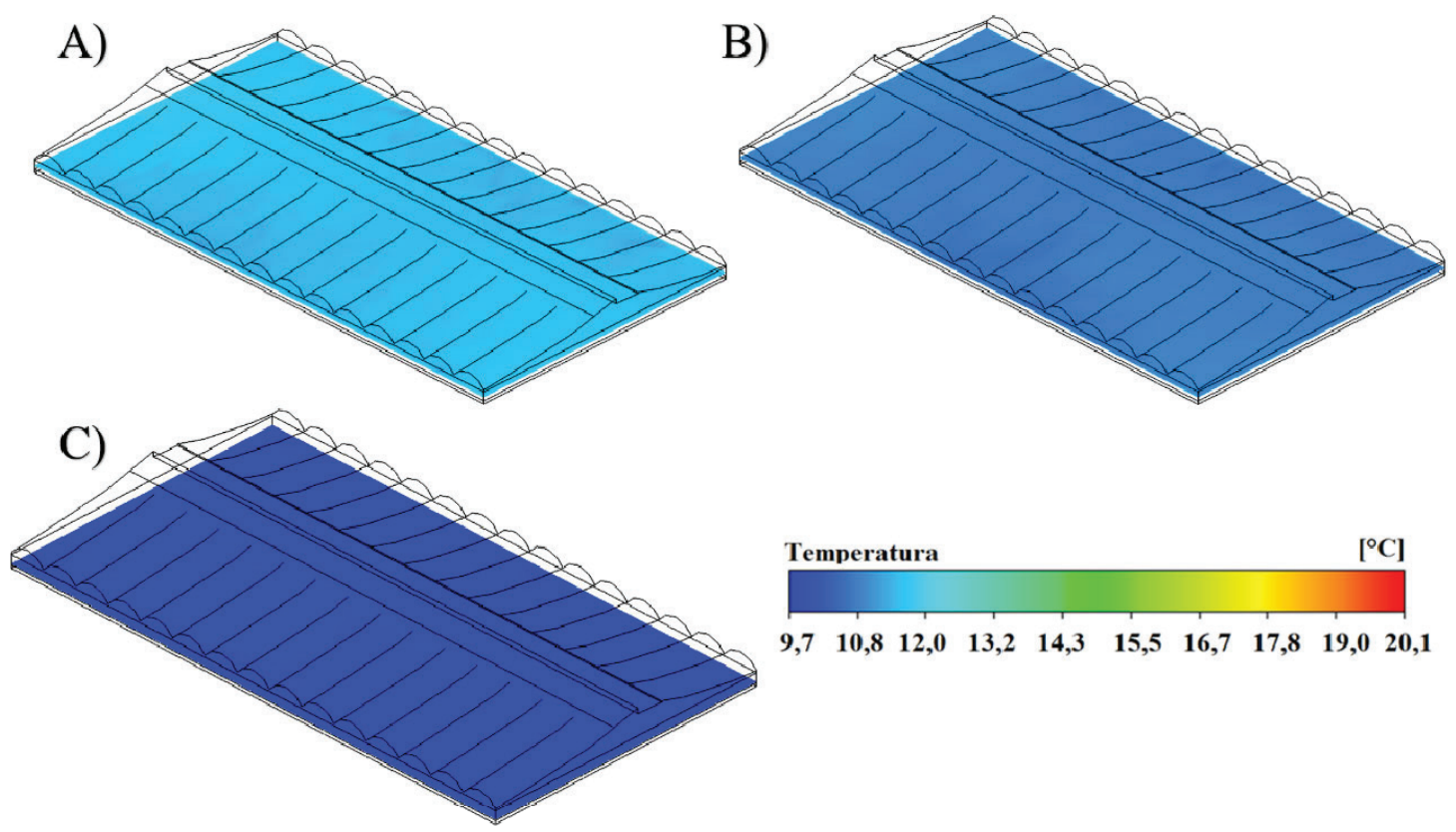

Fig. 7. Contornos de temperatura $\left({ }^{\circ} \mathrm{C}\right)$ simulados para: A) hora 20:00; B) hora 00:00; C) hora 04:00. Fig. 7. Temperature contours $\left({ }^{\circ} \mathrm{C}\right)$ simulated for: $\left.A\right)$ hour 20:00; B) hour 00:00; C) hour 04:00.
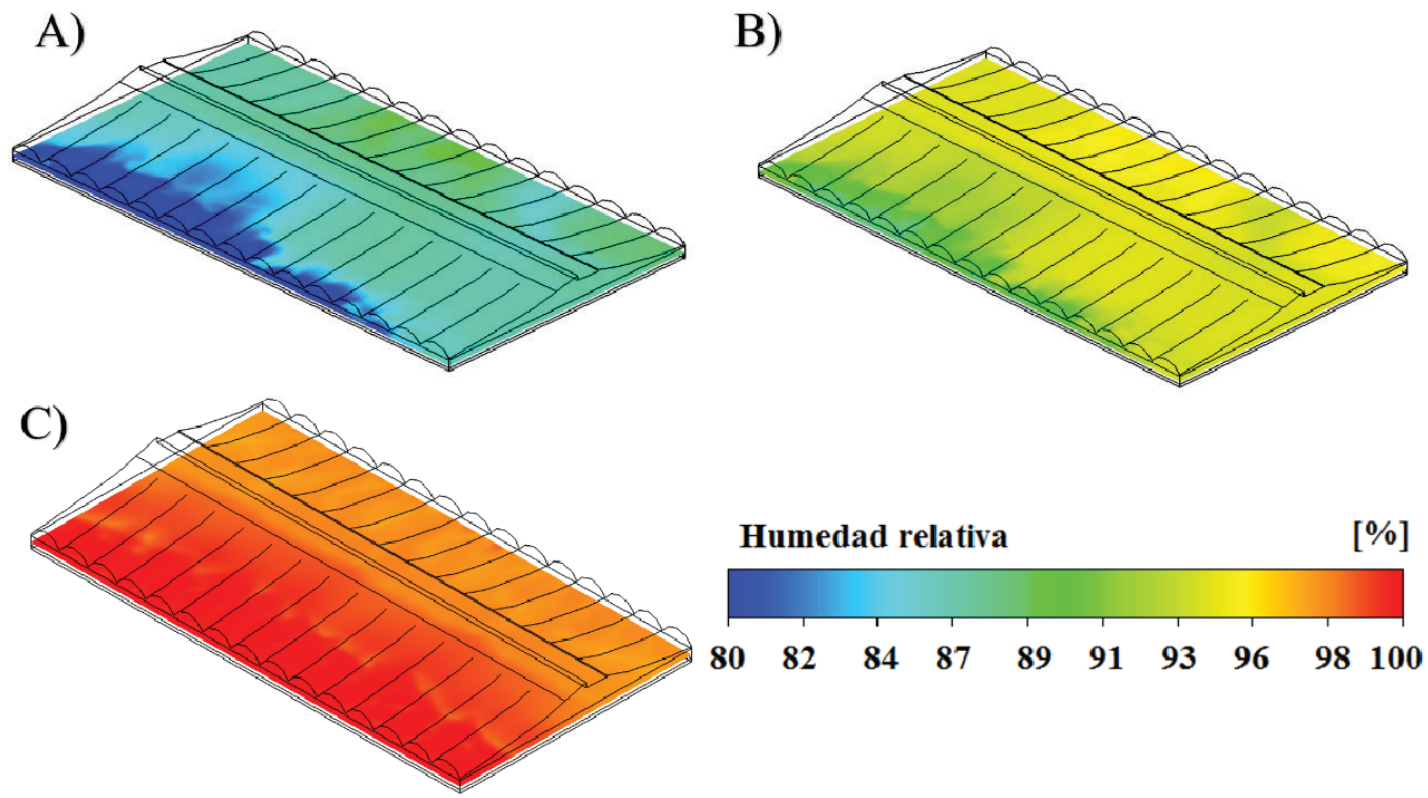

Fig. 8. Contornos de humedad relativa (\%) simulados para: A) hora 20:00; B) hora 00:00; C) hora 04:00. Fig. 8. Relative humidity contours (\%) simulated for: A) hour 20:00; B) hour 00:00; C) hour 04:00. 
respectivamente (Fig. 8A). El valor medio de la HR para la hora 00:00 es igual a $94,1 \%$, con algunas regiones con valores mínimos y máximos de HR de 91 y 96\% (Fig. 8B). Para la hora 04:00 se observa un comportamiento similar con un valor medio de HR de $98,2 \%$.

Los valores para el comportamiento temporal de la HR se presentan en la Tabla 3. En general se puede observar que para el periodo evaluado el valor de $\mathrm{HR}$ es superior al $80 \%$ con una tendencia creciente a medida que trascurre la noche. Para el periodo comprendido entre las 1:00 y 5:00 horas, el valor de la HR presentó valores superiores al $94 \%$, esto es indicativo de un aire altamente saturado lo cual es una característica común del ambiente exterior en la sabana de Bogotá. Esta condición no es adecuada para la producción de rosas, ya que los valores altos de HR en combinación con el fenómeno de inversión térmica y déficit de presión de vapor bajos, suelen generar condensación del vapor de agua en la cubierta del invernadero y en la superficie foliar de las plantas, creando las condiciones favorables para la aparición de enfermedades fúngicas (Baptista et al., 2012). Adicionalmente en los invernaderos pasivos cuando aparece el fenómeno de condensación, éste se extiende hasta las primeras horas del periodo diurno, lo cual tiende a reducir la tasa de transpiración de las plantas, generando desórdenes fisiológicos debido a la translocación de algunos nutrientes como el calcio (Wang et al., 2012).

\section{CONCLUSIONES}

El modelo numérico CFD-3D fue validado mediante la toma de datos experimentales, que permitieron realizar una comparación entre los datos simulados y medidos. Esta comparación mostró que el modelo utilizado tiene una alta capacidad para representar el comportamiento real de las variables estudiadas. El grado de ajuste es adecuado y los errores de simulación están por debajo del $10 \%$.

El análisis desarrollado permitió establecer que el comportamiento de las variables temperatura y HR para el periodo diurno, presentó valores adecuados para la producción de rosas, caso contrario sucedió para el periodo nocturno donde el comportamiento de estas variables mostró valores subóptimos para su producción. Adicionalmente, se observó la presencia frecuente de inversión térmica, fenómeno que, complementado con la presencia de altas humedades relativas, favorece la condensación del vapor de agua, lo cual genera ambientes propicios para el ataque de enfermedades fúngicas y el desarrollo de desórdenes fisiológicos en las plantas. El enfoque de este trabajo puede ser utilizado en investigaciones futuras, aunque se recomienda incluir el cultivo y observar las variaciones que puedan ocasionar las plantas al microclima generado.

\section{RECONOCIMIENTO}

Los autores agradecen al Servicio Nacional de Aprendizaje (SENA), la Asociación Colombiana de Exportadores de Flores (Asocolflores) y al Centro de Innovación de la Floricultura Colombiana (Ceniflores).

Conflicto de intereses: El manuscrito fue preparado y revisado con la participación de todos los autores, quienes declaramos que no existe conflicto.

\section{LITERATURA CITADA}

Ansys Corporation. 2016. ANSYS FLUENT User's Guide v17. 2016. Available at https://www. ansys.com/es-es/academic/free-studentproducts. (Accessed 6 April 2016)

Baeza, E.J., J.J. Pérez-Parra, J.I. Montero, B.J. Bailey, and J.C. López. 2009. Analysis of the role of sidewall vents on buoyancydriven natural ventilation in parral-type greenhouses with and without insect screens using computational fluid dynamics. Biosyst. Eng. 104(1): 86-96. doi: 10.1016/J. BIOSYSTEMSENG.2009.04.008.

Baeza, E.J., J.I. Montero, J. Pérez-Parra, y B.J. Bailey. 2012. Avances en el estudio de la ventilación natural. Disponible en http://www. publicacionescajamar.es/pdf/series-tematicas/ centros-experimentales-las-palmerillas/ avances-en-el-estudio-de-la-ventilacion.pdf (Consulta 21 de mayo de 2018).

Baptista, F.J. 2007. Modelling the climate in unheated tomato greenhouses and predicting Botrytis cinerea infection. 180 p. Thesis Ph.D. of Rural Engineering. Univerisade de Évora, Évora Portugal.

Baptista, F.J., B.J. Bailey, and J.F. Meneses. 2012. Effect of nocturnal ventilation on the occurrence of Botrytis cinerea in Mediterranean unheated tomato greenhouses. Crop Protection 32:144-149.

Bournet, P.E, and T. Boulard. 2010. Effect of ventilator configuration on the distributed climate of greenhouses: A review of experimental and CFD studies. Comput. Electron. Agric. 74 (2010) 195-217. doi:10.1016/j.compag.2010.08.007. 
Baxevanou, C., D. Fidaros, T. Bartzanas, and C. Kittas. 2017. Yearly numerical evaluation of greenhouse cover materials. Computers and Electronics in Agriculture 149:54-70. doi. org/10.1016/j.compag.2017.12.006.

Castillo, C., E. Álvarez, E. Gómez, G. Llano, y J. Zapata. 2010. Mejoramiento nutricional de la rosa para el manejo de Peronospora sparsa Berkeley, causante del mildeo velloso. Rev. Acad. Colomb. Cienc. Exacto. Fis. Nat. 34(131):137-142.

Chen, Q. 2009. Ventilation performance prediction for buildings: A method overview and recent applications. Building and Environment 44(4):848-858. https://doi.org/10.1016/j. buildenv.2008.05.025.

Espinal-Montes, V., I. López-Cruz, A. Rojano-Aguilar, E. Romantchik-Kriuchova, y A. Ramírez-Arias. 2015. Determinación de los gradientes térmicos nocturnos en un invernadero usando dinámica de fluidos computacional. Agrociencia 49(3):233-247.

Flores-Velázquez, J., y F. Villarreal-Guerrero. 2015. Diseño de un sistema de ventilación forzada para un invernadero cenital usando CFD. Revista Mexicana de Ciencias Agrícolas 6(2):303-316.

He, X., J. Wang., S. Guo., J. Zhang., B. Wei., J.Sun., et al. 2017. Ventilation optimization of solar greenhouse with removable back walls based on CFD. Computers and Electronics in Agriculture 149:16-25. doi.org/10.1016/j.compag.2017.10.001.

Iglesias, N., J.I. Montero, P. Muñoz, y A. Antón. 2009. Estudio del clima nocturno y el empleo de doble cubierta de techo como alternativa pasiva para aumentar la temperatura nocturna de los invernaderos utilizando un modelo basado en la mecánica de fluidos computacional (CFD). Hort. Argentina 28:18-23.

Kacira, M., S. Sase., and L. Okushima. 2004. Effects of side vents and span numbers on wind-induced natural ventilation of a gothic multi-span greenhouse. Japan Agricultural Research Quarterly 38(4):227-233. doi. org/10.6090/jarq.38.227.

Kinfe, A. M., B. Vanthoor, B., B.O. Van't. 2014. Effect of greenhouse cover spectral properties on rose yield for Ethiopia highland. International Journal of Innovation and Applied Studies 9(4):1926-1937.

McCartney, L., V. Orsat, and M.G. Lefsrud. 2018. An experimental study of the cooling performance and airflow patterns in a model Natural Ventilation Augmented Cooling (NVAC) greenhouse, Biosyst Eng. 174 (2018) 173-189. doi:10.1016/j.biosystemseng.2018.07.005.
Mena-Vásconez, P., Boelens, R, and J. Vos. 2016. Food or flowers? Contested transformations of community food security and water use priorities under new legal and market regimes in Ecuador's highlands. J. Rural Stud. 44:227-238. doi:10.1016/j.jrurstud.2016.02.011.

Mesmoudi, K., K. Meguallati, and P. Bournet. 2017. Effect of the greenhouse design on the thermal behavior and microclimate distribution in greenhouses installed under semi-arid climate. Heat Transfer. Asian Research 46(8):1294-1311. doi.org/10.1002/ htj.21274.

Montero, J.I. 2006. Evaporative cooling in greenhouses: Effect on microclimate, water use efficiency and plant respons. Acta Horticulturae 719:373-383. doi.org/10.17660/ ActaHortic.2006.719.42.

Montero, J.I. P. Muñoz, M.C. Sánchez-Guerrero, E. Medrano, D. Piscia, and P. Lorenzo. 2013. Shading screens for the improvement of the night time climate of unheated greenhouses. Spanish J. Agric. Res. 11 (2013) 32. doi:10.5424/ sjar/2013111-411-11.

López, A., D.L. Valera, and F. Molina-Aiz. 2011. Sonic anemometry to measure natural ventilation in greenhouses. Sensors 11(10):9820-9838.

Piscia, D., J.I. Montero, E. Baeza, and B.J. Bailey. 2012. A CFD greenhouse night-time condensation model. Biosystems Engineering 111(2):141-154. doi.org/10.1016/j. biosystemseng.2011.11.006.

Piscia, D., P. Muñoz, C. Panadès, and J.I. Montero. 2015. A method of coupling CFD and energy balance simulations to study humidity control in unheated greenhouses. Comput. Electron. Agric. 115:129-141. doi:10.1016/j. compag.2015.05.005.

Restrepo, F. 2010. Manual de manejo de Botrytis cinerea en rosas. 120 p. Ediciones Ceniflores, Bogota, Colombia.

Rico-García. E. 2011. Aerodynamic study of greenhouses using computational fluid dynamics. International Journal of the Physical Sciences 6(28). doi.org/10.5897/ IJPS11.852.

Rojano, F., J. Flores-Velázquez, F. VillarrealGuerrero, y A. Rojano. 2014. Dynamics of climatic conditions in a greenhouse: Two locations in Mexico. Acta Horticulturae 1037:955-962. https://doi.org/10.17660/ ActaHortic.2014.1037.127 
Tominaga, Y., A. Mochida, R. Yoshie, H. Kataoka, T. Nozu, M. Yoshikawa, et al. 2008. AIJ guidelines for practical applications of CFD to pedestrian wind environment around buildings. Journal of Wind Engineering and Industrial Aerodynamics 96(10-11):17491761. doi.org/10.1016/j.jweia.2008.02.058.

Villagrán, E.A., R. Gill., J.F. Acuña., and C.R. Bojacá. 2012. Optimization of ventilation and its effect on the microclimate of a colombian multispan greenhouse. Agronomia Colombiana 30(2):282-288.

Villagran Munar, E., C. Bojacá Aldana., y N. Rojas Bahamon. 2018. Determinación del comportamiento térmico de un invernadero espacial colombiano mediante dinámica de fluidos computacional. Revista U.D.C.A Actualidad \& Divulgación Científica 21(2):415-426. https://doi.org/10.31910/rudca. v21.n2.2018.1070
Wang, X., G. He, G, ans Y. Zhang. 2012. CFD simulation of the temperature and humid distribution for natural ventilated modern greenhouse. In 2012 International Conference on Computer Science and Electronics Engineering Vol. 1. p. 680-683). IEEE.

Yong, A. 2004. El cultivo del rosal y su propagación. Cultivos Tropicales 25(2): 53-67. 